\title{
A Case of Gait Disturbance Resolved with Hysterectomy in a Woman
}

\section{Soo-Ho Chung}

Department of Obstetrics and Gynecology, Soonchunhyang University Bucheon Hospital, Soonchunhyang University College of Medicine, Bucheon, Korea

\begin{abstract}
Uterine leiomyoma is the most common benign tumors in females and varies widely in size. Lumbosacral plexopathy is a rare disorder characterized by a sudden onset of leg paresthesia, muscle weakness, and reduction of deep tendon reflexes. This condition usually caused by compression from an abdominal mass, paralysis in mothers during labor, and compression of the lumbosacral plexus by the fetal head during dystocia. This case report presents a 55-year-old woman with a huge uterine myoma and symptoms of weakness of the right leg and gait disturbance. She was diagnosed with lumbosacral plexopathy that resolved after a laparoscopic-assisted total hysterectomy.
\end{abstract}

Keywords: Lumbosacral plexopathy; Gait disorder; Leiomyoma

\section{INTRODUCTION}

Uterine leiomyoma is the most common benign tumor of the uterus in women of 30 and 45 years and is one leading cause of hysterectomy [1]. Many women with uterine leiomyoma do not experience clinical symptoms, while submucosal leiomyoma may present with multiple symptoms. Uterine leiomyoma varies widely in size, and the largest size has reportedly been $45 \mathrm{~kg}$ [2]. The author reports a case of lumbosacral plexopathy compressed by a uterine myoma.

\section{CASE REPORT}

A 55-year-old woman (G3 L2) who out-patiently visited Soonchunhyang University Bucheon Hospital with major complaints of vaginal bleeding, uterine myoma, and adenomyosis persisted for 2 years after 5 years of menopause was admitted to the department of neurosurgery due to muscle weakness in the right leg and walking difficulty during the last 2 weeks prior to admission. The patient had received aneurysm clipping due to subarachnoid hemorrhage (anterior communicating artery aneurysm) in 2007 and is currently taking medication for high blood pressure (BP) diagnosed in 2013. On admission, she had BP of $140 / 80 \mathrm{~mm} \mathrm{Hg}$, pulse rate 75 beats/min, respiratory rate of 20 beats/min, and body temperature of $36.6^{\circ} \mathrm{C}$. Lower abdominal distension was found and a large hard mass was palpated. Vaginal bleeding was associated with hematomas, and she was unable to bend her right leg and walk. Laboratory findings revealed a hemoglobin level of $8.5 \mathrm{~g} / \mathrm{dL}$, packed cell volume of $26.3 \%$, and platelet count of $236,000 / \mu \mathrm{L}$. She had a normal chest X-ray and electrocardiogram and underwent brain computed tomography (CT), diffusion magnetic resonance imaging, abdominal CT, and electromyography (EMG). Brain exam revealed no finding suspected of walking abnormality (Fig. 1). She underwent total laparoscopic hysterectomy (Fig. 2). The weight of specimen was $870 \mathrm{~g}$, and the pathologic results were leiomyoma and adenomyosis. Since no apparent signs were shown in a previous lesion, a total hysterectomy was performed on the 7th day after admission to the hospital. She was able to ambulate with assistance on the 6th postoperative day and herself independently on the 9 th postoperative day. She was discharged after progression in symptoms. EMG and other tests were conducted at an outpatient visit in the first postoperative month (Table 1). The patient restored the ability to perform the usual activities of daily living. And the patient provided written informed consent for the publication of clinical details and images. 


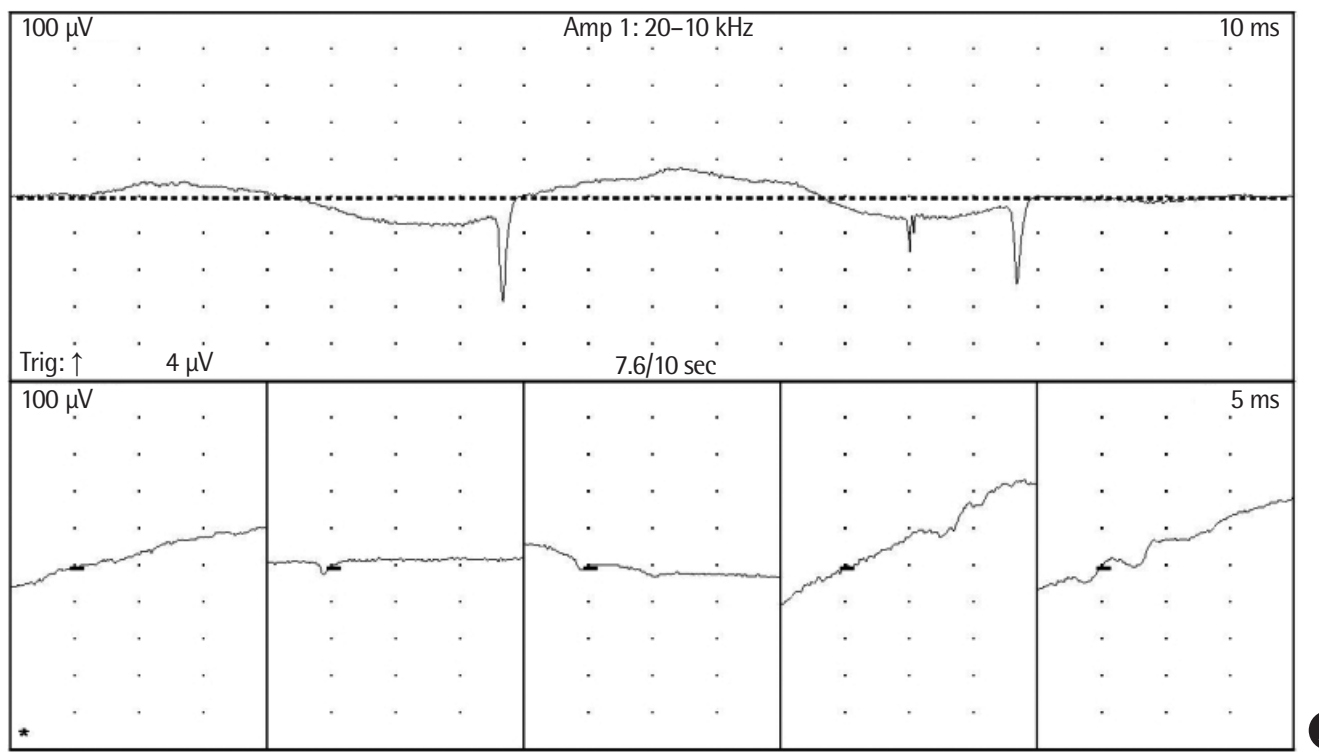

\begin{tabular}{lcccc}
\hline Muscle & Side/site & Insertionalactivity & PSW & FP \\
\hline Tibialis anterior & Right & Normal & None & None \\
Peroneus longus & Right & Normal & None & None \\
Gastrocnemius & Right & Normal & None & None \\
Vastus lateralis & Right & Normal & None & None \\
Rectus femoris & Right & Normal & None & None \\
Biceps femoris & Right & Normal & None & None \\
Gluteus maxius & Right & Normal & None & None \\
L5-S1 & Right & Increased & +2 & None \\
L4 & Right & Increased & +1 & None \\
L3 & Right & Normal & None & None \\
\hline
\end{tabular}

Fig. 1. (A, B) Electrophysiological abnormalities suggesting lumbosacral poly-radiculopathy were observed in the nerve conduction test in both lower limbs and the electromyography in the right lower extremity and trunk. PSW, positive sharp wave; FP, fibrillation potential.
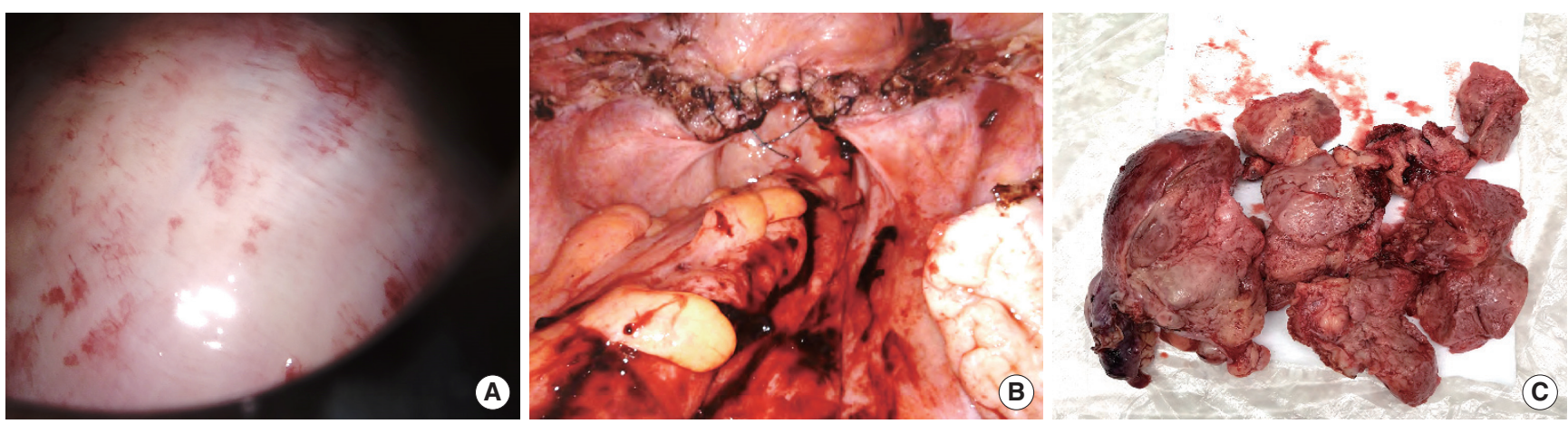

Fig. 2. This pictures shows (A) preoperation, (B) postoperation, and (C) specimen.

\section{DISCUSSION}

The lumbosacral plexus consists of the lumbar plexus formed by $\mathrm{L} 1-\mathrm{L} 4$ nerve roots and the sacral plexus receiving contributions from L4-L5 and S1-S3 nerve roots [3]. The lumbar plexus is located within the psoas major muscle, and its major derivatives are the femoral and the obturator nerves. The sacral plexus is located on the surface of the posterior pelvic wall, and its major branches are 
Chung SH • Gait Disturbance Resolved with Hysterectomy

Table 1. Postoperative electro-physiologic test and electromyography

\begin{tabular}{lcccc}
\hline Muscle & Side/site & Insertional activity & PSW & FP \\
\hline Tibialis anterior & Right & Normal & None & None \\
Gastrocnemius & Right & Normal & None & None \\
Vastus lateralis & Right & Normal & None & None \\
Biceps femoris & Right & Normal & None & None \\
L5-S1 & Right & Normal & None & None \\
\hline
\end{tabular}

Postoperative electro-physiologic test and electromyography was normal. PSW, positive sharp wave; FP, fibrillation potential.

the tibia and common peroneal nerves that travel down from the sciatic nerve [3]. The lumbosacral plexus extends to the organs of the abdomen and pelvis, and lumbosacral plexus palsy can occur secondary to injury associated with another disease in this complex structure [4]. Lumbosacral plexopathy is a rare type of peripheral neuropathy, and peripheral neuropathy is classified into radiculopathy, plexopathy, mononeuropathy, multiple mononeuropathy, and polyneuropathy depending on its application site [5]. Of these, plexopathy can be divided into brachial plexopathy and lumbosacral plexopathy depending on anatomical location of involvement. Lumbosacral plexopathy has been rarely diagnosed in patients [6]. Lumbosacral plexopathy is characterized by a sudden leg paresthesia, muscle weakness, and a reduction of deep tendon reflex [5]. The causes of lumbosacral plexopathy can be caused by penetrating injury, vertebral fracture, pelvic fracture, surgical trauma, and compression from an abdominal mass such as a pelvic tumor, aortic aneurysm or hematoma, paralysis in mothers during labor, and compression of the lumbosacral plexus by the fetal head during dystocia [6]. Occasionally, lumbosacral plexopathy can occur after radiotherapy in carcinoma patients and has been found in patients with cervical cancer after radiotherapy [7]. Other related factors are diabetes, polyarteritis nodosa, infection, and agglutination reaction, and it can also occur due to unknown causes in some cases [8,9]. Lumbosacral plexopathy is not limited to a single nerve root or region but can result in unilateral muscle palsy and changes in sensory function and reflexes [9]. Depending on the affected site, this condition may involve the femoral, obturator, and sciatic nerves and incur motor or sensory paralysis [9]. The patient was initially thought to be a gait disorder due to aneurysm clipping, but there was no abnormality in brain imaging. She was later diagnosed as having a gait disorder caused by compression of the large uterus in abdominal cavity. Symptoms can be unilateral or bilateral involvement depending on the causes, and aggravate to muscle weakness, sensory loss, loss of reflex and pain
Table 2. Characteristics of nerve compression patients due to uterine myoma

\begin{tabular}{llrl}
\hline & \multicolumn{1}{c}{ Main symptom } & $\begin{array}{c}\text { Uterus } \\
\text { weight (g) }\end{array}$ & \multicolumn{1}{c}{ Operation } \\
\hline Case 1 & Gait disturbance & 870 & Laparoscopic hysterectomy \\
Case 2 & Right side foot drop & 2,200 & Abdominal hysterectomy \\
Case 3 & Radiating pain in lower limb & 1,000 & Abdominal hysterectomy \\
\hline
\end{tabular}

of burning sensation, cough, sneezing, and others [6]. Typically, upper-plexus lesions may cause weakness of the femur and tarsus and sensory loss in the back of the leg, while lower plexus lesions may result in weakness of hip flexion and adduction and sensory loss in the front of the leg [6]. EMG of the lesions of the nervous system is a relatively objective and accurate examination test that is helpful in differential diagnosis to determine the location and degree of peripheral nerve injury [6]. For accurate assessment of injuries of the lumbosacral plexus involved, extensive techniques have been introduced including nerve conduction study and needle EMG partially screening the femoral and sciatic nerves and their branches, $\mathrm{H}$ reflexes, F waves, somatosensory evoked potential, and others [10]. In this case, the gait disorder could not be diagnosed with other radiology results, but the diagnosis and treatment effect was confirmed through EMG. Uterine leiomyoma is the most common benign tumors occurring in the female pelvis and affect about $20 \%$ of women of childbearing age and $40 \%-50 \%$ of women aged more than 35 years, and the clinical symptoms are present in about $20 \%-50 \%$ of the affected women and include a self-palpated mass, uterine bleeding, acute or chronic pelvic pain, and compression [1]. In this case report, the patient had muscle weakness and gait impairment suspected to be caused by compression on the lumbosacral nerves due to a giant uterine leiomyoma. EMG revealed positive sharp waves of 2+ and 1+ in L5-S1 and L4 (Fig. 1). In this case, laparoscopic-assisted total hysterectomy was performed. She was able to ambulate with assistance on the 6 th postoperative day and herself independently on the 9th postoperative day. The improved outcome was shown in postoperative EMG compared to preoperative EMG (Table 1), and she is currently able to perform the usual activities of daily living. In medical literature search, lumbosacral plexopathy due to a huge uterine myoma has been reported in only three cases, and all patients improved after hysterectomy (Table 2) [9]. Even as an obstetrician/ gynecologist, lumbosacral plexopathy is a rare and unfamiliar disorder, and it is not easy to predict that paresthesia and weakness of the legs and gait disturbance can be caused by compression due to 
a large uterine myoma [11]. We report a case of gait disturbance due to compression of the lumbosacral plexus caused by uterine myoma and recovery after hysterectomy.

\section{REFERENCES}

1. Walid MS, Heaton RL. Laparoscopic myomectomy: an intent-to-treat study. Arch Gynecol Obstet 2010;281:645-9.

2. Song T, Kim TJ, Kim MK, Park H, Kim JS, Lee YY, et al. Single port access laparoscopic-assisted vaginal hysterectomy for large uterus weighing exceeding 500 grams: technique and initial report. J Minim Invasive Gynecol 2010;17:456-60.

3. Alsever JD. Lumbosacral plexopathy after gynecologic surgery: case report and review of the literature. Am J Obstet Gynecol 1996;174:1769-78.

4. Warner MA, Martin JT, Schroeder DR, Offord KP, Chute CG. Lower-extremity motor neuropathy associated with surgery performed on patients in a lithotomy position. Anesthesiology 1994;81:6-12.

5. Yee T. Recurrent idiopathic lumbosacral plexopathy. Muscle Nerve 2000; 23:1439-42.

6. Van Alfen N, Malessy MJ. Diagnosis of brachial and lumbosacral plexus lesions. Handb Clin Neurol 2013;115:293-310.

7. Gonzalez-Caballero G, Arroyo-Gonzalez R, Vazquez-Perez AV, de las Heras V, Gonzalez-Gutierrez JL. Lumbosacral plexopathy 15 years after radiotherapy for carcinoma of the cervix. Rev Neurol 2000;30:97.

8. Ishii K, Tamaoka A, Shoji S. MRI of idiopathic lumbosacral plexopathy. Neurology 2004;63:E6.

9. Dyck PJ, Thaisetthawatkul P. Lumbosacral plexopathy. Continuum (Minneap Minn) 2014;20(5 Peripheral Nervous System Disorders):1343-58.

10. Ko HY, Kim KT. Treatment of external urethral sphincter hypertonicity by pudendal nerve block using phenol solution in patients with spinal cord injury. Spinal Cord 1997;35:690-3.

11. Barnett JC, Hurd WW, Rogers RM Jr, Williams NL, Shapiro SA. Laparoscopic positioning and nerve injuries. J Minim Invasive Gynecol 2007;14: 664-72. 